\title{
EXTENDED CLUSTERING COEFFICIENTS:GENERALIZATION OF CLUSTERING COEFFICIENTS IN SMALL-WORLD NETWORKS *
}

\author{
Wenjun XIAO $^{1}$, Wenhong WEI ${ }^{1}$, Weidong CHEN ${ }^{1}$, Yong QIN ${ }^{1}$, Behrooz PARHAMI ${ }^{2}$ \\ ${ }^{I}$ Department of Computer Science, South China University of Technology, \\ Guangzhou 510641, China \\ wjxiao@scut.edu.cn (凹) \\ ${ }^{2}$ Department of Electrical \& Computer Engineering, University of California, \\ Santa Barbara, CA 93106-9560, USA
}

\begin{abstract}
The clustering coefficient $C$ of a network, which is a measure of direct connectivity between neighbors of the various nodes, ranges from 0 (for no connectivity) to 1 (for full connectivity). We define extended clustering coefficients $C(h)$ of a small-world network based on nodes that are at distance $h$ from a source node, thus generalizing distance-1 neighborhoods employed in computing the ordinary clustering coefficient $C=C(1)$. Based on known results about the distance distribution $P_{\delta}(h)$ in a network, that is, the probability that a randomly chosen pair of vertices have distance $h$, we derive and experimentally validate the law $P_{\delta}(h) C(h) \leq c \log N / N$, where $c$ is a small constant that seldom exceeds 1 . This result is significant because it shows that the product $P_{\delta}(h) C(h)$ is upper-bounded by a value that is considerably smaller than the product of maximum values for $P_{\delta}(h)$ and $C(h)$. Extended clustering coefficients and laws that govern them offer new insights into the structure of small-world networks and open up avenues for further exploration of their properties.
\end{abstract}

Keywords: Clustering coefficient, small-world, extended clustering coefficient, distance distribution

\section{Introduction}

Complex networks describe a wide range of systems in nature and society. They have recently attracted considerable interest because the complex networks are powerful models for unified analysis of a wide range of systems in society and nature. Complex networks are currently being studied across many fields of science (Barabási \& Albert, 1999, Watts \& Strogatz, 1998, Milgram, 1967, Newman, 2003). Undoubtedly, many systems in nature can be described by models of complex networks, which are structures consisting of nodes or vertices connected by links or edges. Examples are numerous. The Internet is a network of routers or domains. The World Wide Web

\footnotetext{
* This work was supported in part by the Natural Science Foundation of Guangdong Province under Grant No. 04020130. The original version was presented on the International Conference on Computational Science (ICCS 2007)
} 
(WWW) is a network of websites. The brain is a network of neurons. An organization is a network of people. The global economy is a network of national economies, which are themselves networks of markets; and markets are themselves networks of interacting producers and consumers. Food webs and metabolic pathways can all be represented by networks, as can the relationships among words in a language, and topics in a conversation. Moreover, diseases are transmitted through social networks; and computer viruses occasionally spread through the Internet. Energy is distributed through transportation networks, both in living organisms, man-made infrastructures, and in many physical systems such as the power grids. The study of networks, in the form of mathematical graph theory, is one of the fundamental pillars of discrete mathematics, and during the twentieth century graph theory has developed into a substantial body of knowledge. So a network can look as a graph $G=(V, E)$ which has a set $V$ of $N$ vertices or nodes and a set $E$ of $M$ edges or links, where each edge is defined by a pair of vertices (ordered pair, for directed graphs) (Newman, 2003). Actually, a set of vertices and edges only construct the simplest type of network, but there are many constructing ways in which networks may be more complex than this. For instance, there may be more than one different type of vertex in a network, or more than one different type of edge. And vertices or edges may have a variety of properties, numerical or otherwise, associated with them. Taking the example of a social network of people, the vertices may represent men or women, people of different nationalities, locations, ages, incomes, or many other things. Edges may represent friendship, but they could also represent animosity, or professional acquaintance, or geographical proximity. They can carry weights, representing, say, how well two people know each other. They can also be directed, pointing in only one direction. Graphs composed of directed edges are themselves called directed graphs or sometimes digraphs, for short. A graph representing telephone calls or email messages between individuals would be directed, since each message goes in only one direction. Directed graphs can be either cyclic, meaning they contain closed loops of edges, or acyclic meaning they do not. Some networks, such as food webs, are approximately but not perfectly acyclic.

Two models of actual complex networks have been studied extensively: the small-world model and the scale-free one. Our focus in this paper is on small-world networks that feature localized clusters connected by occasional long-range links, leading to an average distance between vertices that grow logarithmically with the network size $N$.

The small-world phenomenon comes from the observation that individuals are often linked by a short chain of acquaintances. The small-world concept in simple terms describes the fact that despite their often large size, in most networks there is a relatively short path between any two nodes. The distance between two nodes is defined as the number of edges along the shortest path connecting them. Milgram (1967) conducted a series of mail delivery experiments and found that an average of 'six degrees of separation' exists between senders and receivers. Small worlds were also 
observed in the context of the Internet and the World Wide Web. To understand network structures that exhibit low degrees of separation, Watts and Strogatz (1998) conducted a set of rewiring experiments on networks, and observed that by rewiring a few random links in regular graphs, the average path length was reduced drastically (approaching that of random networks), while the clustering 1 remains almost constant (similar to that of regular networks). This class of networks was termed small-world networks, and it emphasizes the importance of random links acting as short-cuts that contract the average path length of the network.

Watts and Strogatz (1998) studied mechanisms via which a regular network can be transformed into a small-world network, with little or no change in the vertex-degree distribution, and quantified the parameters that characterize the resulting structures. Clustering is a main characterize of small-world network, for example, in social networks, a common property of is that cliques form, representing circles of friends or acquaintances in which every member knows every other member. This inherent tendency to cluster is quantified by the clustering coefficient (Strogatz, 1998), a concept that has its roots in sociology, appearing under the name 'fraction of transitive triples' (Milgram, 1967). The definition of clustering coefficient will be introduced in the follow.

The paper is organized as follows: In Section 2 we give some preliminary knowledge including descriptions of the Cayley graph network model, the deterministic small-world model and clustering coefficient. In Section 3 we present the definition of extended clustering coefficient. In Section 4, we describe the preliminary simulation results for parameter in complex network. In section 5, some formularies and deduction validate the correction of extended clustering coefficient in deterministic small-world model and Section 6 concludes the paper.

\section{Related Work}

\subsection{Cayley Graph Network Models}

It is known that Cayley graphs are excellent models for interconnection networks (Akers \& Krishnamurthy, 1989). Many well-known and practically useful interconnection networks are Cayley graphs. For example, hypercube and cube-connected cycle networks are Cayley graphs. In Xiao \& Parhami (2006), we know that Cayley graphs can also serve as excellent models for small-world networks.

Let $G$ be a finite group and $S$ a subset of $G$. The subset $S$ is said to be a generating set for $G$, and the elements of $S$ are called generators of $G$, if every element of $G$ can be expressed as a finite product of the powers of the elements in $S$. In this case, we also say that $G$ is generated by $S$. The Cayley digraph of a group $G$ and the subset $S$ of $G$, denoted by $\operatorname{Cay}(G, S)$, has the vertex set that is $G$ and the arc set that is $\{(g, g s) \mid g \in G, s$ $\in S\}$. If $S$ is a generating set of $G$, then we say that $\operatorname{Cay}(G, S)$ is the Cayley digraph of $G$ generated by $S$. If $1 \notin S$ ( 1 is the identity element of $G$ ) and $S=S^{-1}$, then $\operatorname{Cay}(G, S)$ is a simple (undirected) graph. For more definitions and basic results on graphs and groups we refer the reader to books on algebraic graph theory (Biggs, 1993). Unless noted otherwise, all graphs in the following are undirected graphs. 


\subsection{Clustering Coefficient}

One feature shared by small-world networks is that their clustering coefficients are fairly high compared with random networks (Watts \& Strogatz, 1998). Clustering coefficient is defined as follows. Let a vertex $v$ of $G$ have $k(v)$ neighbors; that is, $v$ has degree $k(v)$. These $k(v)$ neighbors can potentially be connected via $k(v)$ $(k(v)-1) / 2$ edges. The fraction of this maximum possible number of edges that actually exist between neighbors of $v$ is its clustering coefficient $C_{v}$; the average of clustering coefficients over all $v \in V$ is the clustering coefficient $C$ of the network $G$. A network with $C$ close to 1 may consist of highly connected clusters or cliques, perhaps with sparser connections between the local clusters.

\subsection{Deterministic Small-World Models}

A great deal of previous research on small-world networks has been based on probabilistic methods, with a rather small number of researchers advocating deterministic models. The Cayley graph model used in our formulation has pedagogical value and can also be used for designing and analyzing communication and the other real networks. To understand the formulation of Cayley graph model, the reader needs a knowledge of elementary group theory (Biggs, 1993). To follow standard notation of group theory, where $G$ stands for a group, we use $\Gamma$ to denote a graph.

Let $G$ be a finite group. Assume that $\Gamma=$ $\operatorname{Cay}(G, S)$ for some generating set $S$ of $G$, where $1 \notin S$ and $S=S^{-1}$. Then $\Gamma$ is a Cayley graph of constant degree $d=|S|$. Thus, every vertex $v$ of $\Gamma$ has exactly $d$ neighbors. Because $\Gamma$ is symmetric, the clustering coefficient of every vertex is the same. Thus, we only need to consider the clustering coefficient of the vertex 1 , the identity element of $G$. The set of neighbors of the vertex 1 is $S$. If $s_{1}, s_{2} \in S$, then $s_{1}$ and $s_{2}$ are adjacent if and only if there is $s \in S$ such that $s_{2}=s_{1} s$. Assume that $H \subseteq S$ and $H \cup 1$ is a subgroup of $G$. Then $s_{1} s_{2} \in H$ when $s_{1}, s_{2} \in H$. Hence, there are at least $|H|(|H|-1) / 2$ edges among the set $S$ of neighbors of the vertex 1 . As a result, the clustering coefficient of $\Gamma$ will be large if $H$ can be chosen to be large. In contrast to the method of Watts and Strogatz (1998), we start from networks with small clustering coefficient and small average distance and proceed to increase the clustering coefficient by means of adding elements of $G$ into $S$, while ensuring that the average distance remains small. In the following we consider an example.

Let $G=Z_{2}^{t}$ be an elementary commutative group of order $2^{t}$. Consider an integer $l$ satisfying $1 \leq l \leq t$. Define $H=\left\{\left(x_{1}, x_{2}, \ldots, x_{l}\right.\right.$, $\left.\left.0^{t-l}\right) \mid\left(x_{1}, x_{2}, \ldots, x_{l}\right) \in Z_{2}^{t}\right\} \backslash\left\{0^{t}\right\}$, where $0^{t}$ is the identity element of $G$ and $S=H \cup\left\{\left(x_{1}\right.\right.$, $\left.x_{2}, \ldots, x_{t}\right) \in Z_{2}^{t} \mid$ only one of $x_{1}, x_{2}, \ldots, x_{t}$ is 1$\}$. Assume that $\Gamma=\operatorname{Cay}(G, S)$. One can easily verify that $|H|=2^{l}-1$ and $|S|=2^{l}+t-l-1$. Hence we obtain that the clustering coefficient of $\Gamma$ is

$$
C=\frac{\left(2^{l}-1\right)\left(2^{l}-2\right)}{\left(2^{l}+t-l-1\right)\left(2^{l}+t-l-2\right)}
$$

Let the diameter (maximum distance between any pair of vertices) of $\Gamma$ be $D(I)$ and $N=2^{t}$. Then $|G|=N$ and $D(\Gamma)=t-l+1 \leq t=$ $\log _{2} N$. Thus, the average distance of $\Gamma$ is no greater than $\log _{2} N$. Based on the result above, we can choose the value of $l$ such that the 
clustering coefficient $C$ is large and the degree of $\Gamma$ is still small. For instance, let $a=\left(2^{l}-1\right) / t$ be a constant. Substituting this value in equation (S1) leads to equation (2) of our paper

$$
C=\frac{a t(a t-1)}{(a t+t-l)(a t+t-l-1)}
$$

As noted in the paper, $C \rightarrow a^{2} /(a+1)^{2}$ when $t \rightarrow \infty$. By suitably choosing $a$, we can obtain different clustering coefficient for $\Gamma$, while maintaining a small vertex degree equal to $a t+t-1=(a+1) \log _{2} N-1$.

One can choose $S$ and $H$ as subsets of $G$ in many different ways, leading to diverse small-world network models. One can further add edges into $\Gamma$ randomly and so obtain models of different real complex networks.

\section{Extended Clustering Coefficients}

Based on the definition of clustering coefficient, we extend the clustering coefficient of Watts and Strogatz (1998) in the following way. Define the $h$-neighbors of a vertex $v$ as vertices of $G$ that are at distance $h$ (measured in number of hops) from $v$. Assume that $v$ has $k_{h}(v)$ such $h$-neighbors, where $k_{1}(v)$ is the same as $k(v)$ defined earlier (see Section 1). Then there can be at most $k_{h}(v)\left(k_{h}(v)-1\right) / 2$ edges connecting $h$-neighbors of vertex $v$. The fraction $C_{v}(h)$ of allowable edges that actually exist between $h$-neighbors $v$ is the $h$-clustering coefficient of $v$. We assume that $C_{v}(h)=1$ when $k_{h}(v)=1$, which also covers the special case $h=0$. The average of $C_{v}(h)$ over all $v \in G$ is the $h$-clustering coefficient $C(h)$ of $G$. The 1-clustering coefficient $C(1)$ is the clustering coefficient $C$ as defined in Section 1.

Thus, while the definition of clustering coefficient is based on the immediate neighborhood of vertices, extended clustering coefficient relates to a wider neighborhood defined by the distance parameter $h$. Using experimental data from a wide variety of actual complex networks, along with a deterministic model of small-world networks that we have developed, we seek to relate $C(h)$ and the distance distribution $P_{\delta}(h)$ of a network, defined as the probability that a randomly chosen pair of vertices are at distance $h$ from each other.

Note that all distances referred to in this paper are shortest distances. However, in view of the results of Kim et al. (2002), distances obtained from a routing algorithm with localized decisions are not fundamentally different from shortest distances in complex networks. Thus, our results are expected to remain valid when this latter definition of distance is used in lieu of shortest distance.

\section{Experimental Analysis}

We start our experiments by investigating several small-world graphs such as NCSTRL graph, Linux graph and Silwood graph. In our experiments, data sets used for these experiments can be seen in appendix. For an $N$-vertex network with $M$ edges, we have $P_{\delta}(0)$ $=1 / N$ and $P_{\delta}(1)=2 M / N^{2}>1 / N$. Beyond $h=1$, however, a precise expression for the value of $P_{\delta}(h)$ cannot be supplied, except in the case of certain regular networks. However, for many networks (small-world or otherwise), the value of $P_{\delta}(h)$ rises with $h$ until it reaches a maximum value and then declines as the distance $h$ gets closer to the network diameter $D$. This is confirmed experimentally for several complex networks of practical interest in Figures $1 b, 2 b$, and $3 b$. 
For extended clustering coefficients, the trend begins by a decrease in clustering, from $C(0)=1$ to $C(1)=C$, and is then followed by further reductions. This is owing to the fact that as $h$ increases, the number $q_{h}$ of nodes at distance $h$ from a given node increases, and such nodes are apt to belong to several cliques; hence, the presence of many edges between them is quite unlikely. As $h$ approaches $D$, however, a different effect may take hold. Consider, for example, one extreme case where each node in the network is at distance $D$ from exactly one node (it has a single diametrically opposite node). This leads to $C(D)=1$. In this same situation, $C(D-1)$ is likely to be large as well, given the common presence of multiple diametral paths to the same opposite vertex. Note that the preceding argument suggests that $C(h)$ can be large when $h$ approaches $D$; it does not imply that $C(h)$ must be large in this case. Figures $1 \mathrm{c}, 2 \mathrm{c}$, and $3 \mathrm{c}$ confirm these trends.

Given the opposing trends of $P_{\delta}(h)$ (up, then down) and $C(h)$ (down, then possibly up), one might be led to believe that the product $P_{\delta}(h)$ $C(h)$ has an upper bound. Based on the evidence presented in Figures 1a, 2a, and 3a, we conjecture that this is in fact the case. That is, for a constant $c$ in the vicinity of and seldom exceeding 1 , we have:

$$
P_{\delta}(h) C(h) \leq c \log N / N
$$

In the special case of $h=1$, equation (1) implies $P_{\delta}(1) C(1) \approx \log N / N$. We have $P_{\delta}(1)=$ $2 M / N^{2} \approx \log N / N$ for small-world networks. This is consistent with $C(1)=C$ being large for such networks.

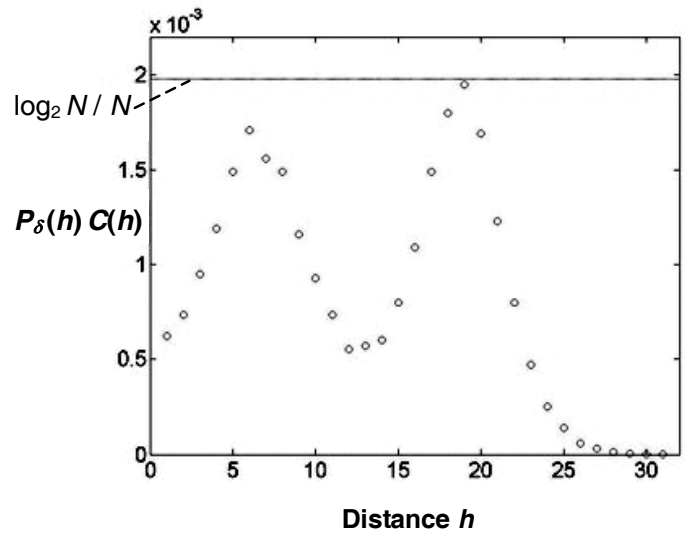

Figure 1a The plot of $P_{\delta}(h) C(h)$ versus $h$ in the maximum component of NCSTRL graph (Newman, 2001), with 6396 vertices and diameter of 31

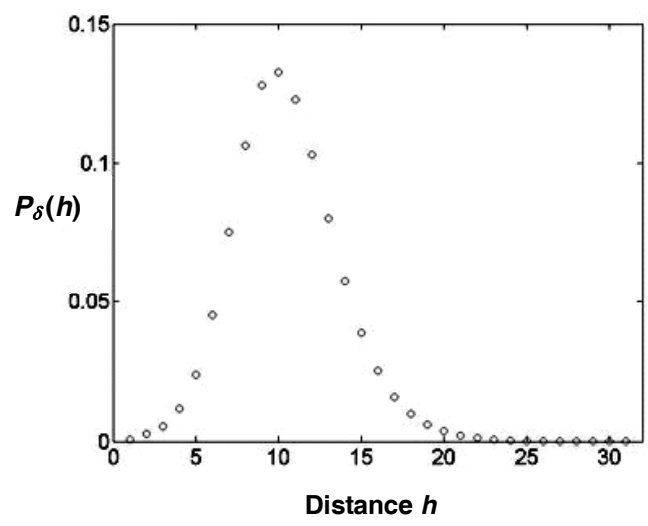

Figure $1 \mathrm{~b}$ The plot of $P_{\delta}(h)$ versus $h$ in $\Delta$

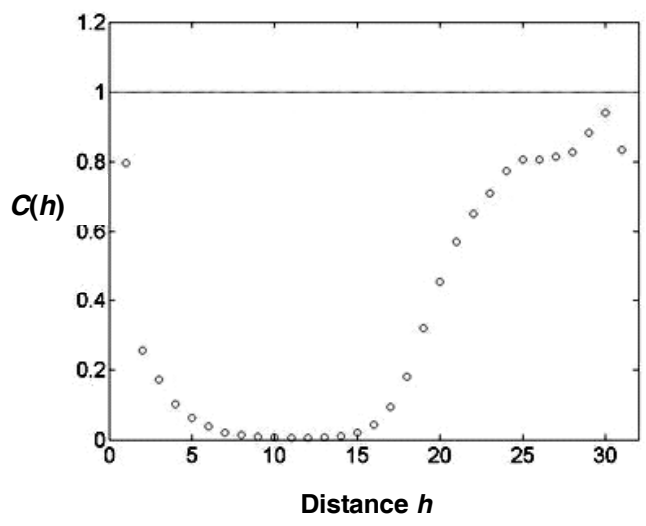

Figure 1c The plot of $C(h)$ versus $h$ in $\Delta$ 


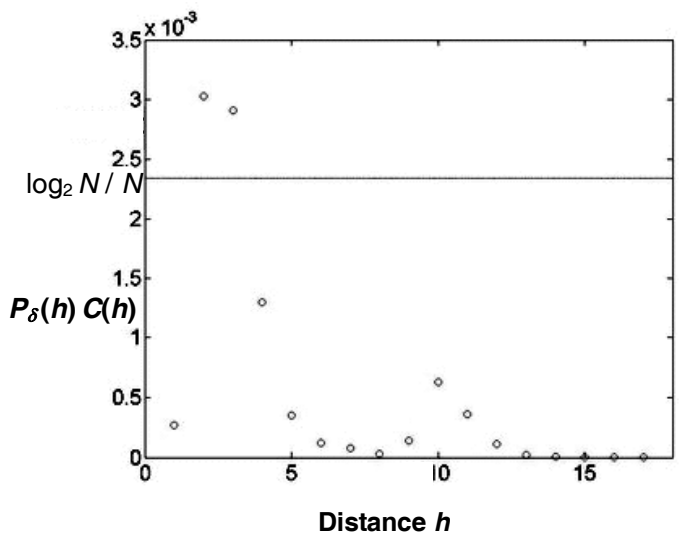

Figure 2a The plot of $P_{\delta}(h) C(h)$ versus $h$ in the maximum component $\Delta_{1}$ of Linux graph (Myers, 2003) with 5285 vertices and diameter of 17 . See http://www.tc.cornell.edu/ myers/Data/SoftwareGraph s/index.html

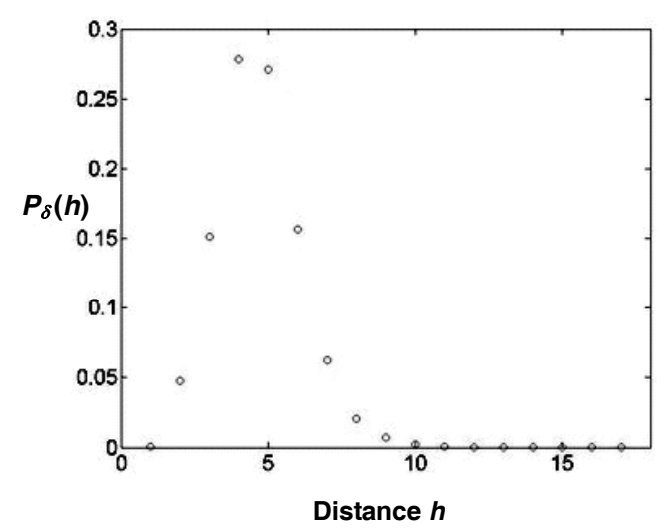

Figure $\mathbf{2 b}$ The plot of $P_{\delta}(h)$ versus $h$ in $\Delta_{1}$

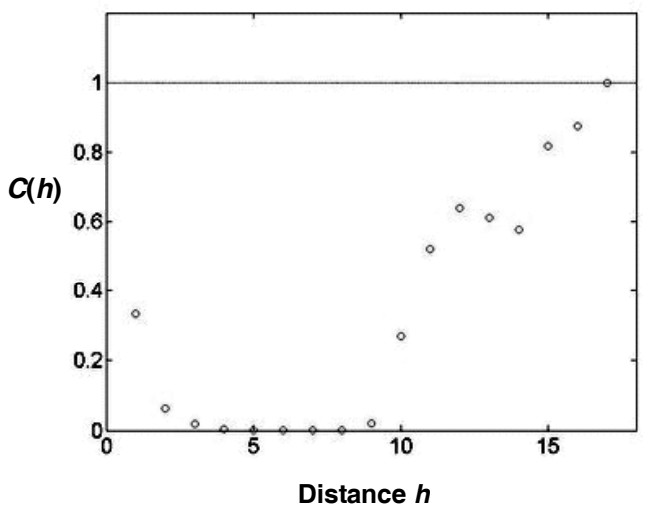

Figure $2 c$ The plot of $C(h)$ versus $h$ in $\Delta_{1}$

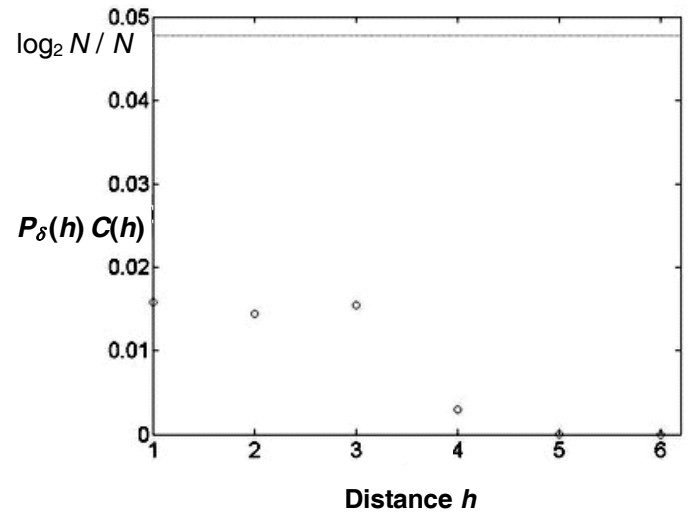

Figure 3a The plot of $P_{\delta}(h) C(h)$ versus $h$ in the maximum component $\Delta_{2}$ of Silwood graph (Montoya \& Sole, 2002) with 153 vertices and diameter of 6 . See http://www.cosin.org/extra/data

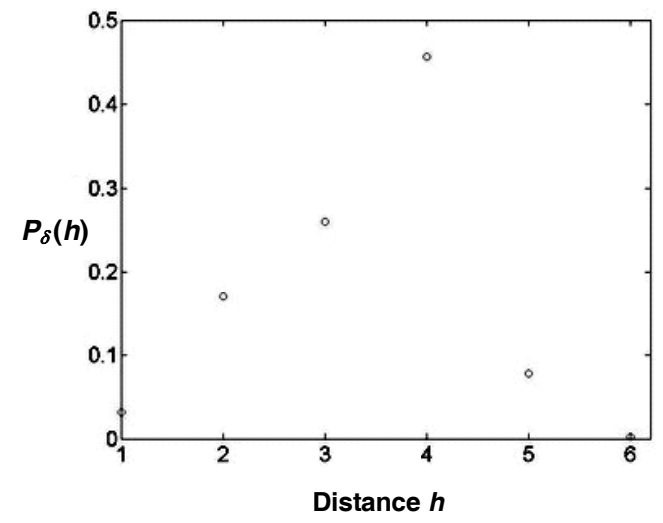

Figure $\mathbf{3 b}$ The plot of $P_{\delta}(h)$ versus $h$ in $\Delta_{2}$

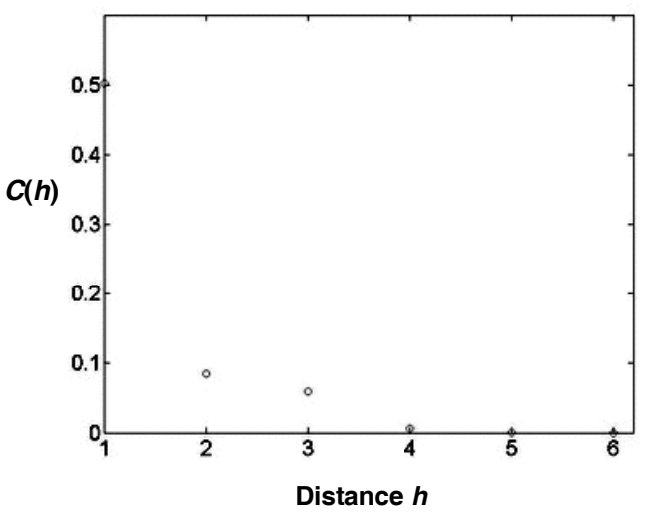

Figure $3 \mathrm{c}$ The plot of $C(h)$ versus $h$ in $\Delta_{2}$ 


\section{Model-Based Validation}

We now present additional evidence on the validity of equation (1), using a model of deterministic small-world networks that we have developed (Xiao \& Parhami, 2006). In fact, it was through this model that we first became aware of the trend represented in equation (1) and experimentally confirmed in Figure 1-3. A review of our deterministic model, which is based on Cayley graphs (Biggs, 1993), has been provided above, where we also show that the model yields the clustering coefficient:

$$
C=\frac{a t(a t-1)}{(a t+t-l)(a t+t-l-1)}
$$

In this model, $t=\log _{2} N$ and $a=\left(2^{l}-1\right) / t$ is a free tuning parameter that is related to the interconnection density, thereby affecting the value of $C$. Note that for very large networks $(N$, $t \rightarrow \infty), C$ tends to $a^{2} /(a+1)^{2}$ when $a$ is a constant. By suitably choosing $a$, we can obtain different clustering coefficients, while maintaining a small vertex degree equal to $a t+t$ $-1=(a+1) \log _{2} N-1$.

Unlike actual networks for which the computation of $C(h)$ is extremely difficult, our deterministic model is amenable to mathematical analysis that yields an approximate closed-form expression for the extended clustering coefficients. In our deterministic model, the number $m$ of adjacent vertex pairs among the $h$-neighbors of any vertex is given by the expression:

$$
m=\left(2^{l}-1\right)\left(2^{l-1}-1\right)\left(\begin{array}{l}
t-l \\
h-1
\end{array}\right)
$$

On the other hand, the number $k_{h}(v)$ of $h$-neighbors of a vertex $v$ is bounded as:

$$
\left(2^{l}-1\right)\left(\begin{array}{c}
t-l \\
h-1
\end{array}\right) \leq k_{h}(v) \leq\left(2^{l}-1\right)\left(\begin{array}{c}
t-l \\
h-1
\end{array}\right)+\left(\begin{array}{c}
t-l \\
h
\end{array}\right)
$$

Given that the extended clustering coefficient $C(h)$ is proportional to $m /\left(k_{h}(v)\right)^{2}$, we readily find:

$$
C(h) \approx 1 /\left(\begin{array}{l}
t-l \\
h-1
\end{array}\right)
$$

In a companion paper (Xiao \& Parhami, 2005), we have derived the distance distribution for small-world networks:

$$
P_{\delta}(h) \approx\left(\begin{array}{c}
\log N \\
h
\end{array}\right) / N
$$

Here, we have $\log N \approx D$. Because the diameter of our deterministic network model is $D=t-l+$ 1, we conclude:

$$
P_{\delta}(h) \approx\left(\begin{array}{c}
t-l+1 \\
h
\end{array}\right) / N=(t-l+1)\left(\begin{array}{c}
t-l \\
h-1
\end{array}\right) /(h N)
$$

Equations (5) and (7) lead to:

$$
P_{\delta}(h) C(h) \leq c \log N / N
$$

Equation (8) confirms our hypothesis in equation (1), thereby supplementing the previously supplied experimental evidence of its validity.

\section{Conclusion}

We have shown that extended clustering coefficients are generalizations of ordinary clustering coefficient and are governed by laws that are also generalizations of those pertaining to the latter. We have presented experimental and analytical evidence that the inequality $P_{\delta}(h) C(h) \leq c \log N / N$ holds for small-world networks. This result is significant because it shows that the product $P_{\delta}(h) C(h)$ is upper-bounded by a value that is considerably 
smaller than the product of maximum values for $P_{\delta}(h)$ and $C(h)$. Thus, extended clustering coefficients offer new insights into the structure of small-world networks and open up further avenues for exploration of their properties. Additionally, different shapes for the variations of $C(h)$ and $P_{\delta}(h) C(h)$, exemplified by Figure $1-3$, can be used to categorize small-world networks in order to facilitate their study.

\section{Acknowledgments}

The authors thank M. E. J. Newman for providing the NCSTRL data used in Figure 1. Authors thank referees for their valuable comments to improve the quality of the paper.

\section{Appendix:}

\section{Data Sets Used for Experimental Results}

For the data on software graphs, see

http://ww.tc.cornell.edu/ myers/Data/SoftwareG raphs/index.html

For the data on biological graphs, see http://www.cosin.org/extra/data

\begin{tabular}{|c|c|c|c|c|c|c|c|c|c|}
\hline Network & \multicolumn{3}{|c|}{ grassland } & \multicolumn{3}{|c|}{ littlerock } & \multicolumn{3}{|c|}{ silwood } \\
\hline \multirow{2}{*}{ Vertices } & $N$ & \multicolumn{2}{|c|}{$\left(\log _{2} N\right) / N$} & $N$ & \multicolumn{2}{|c|}{$\left(\log _{2} N\right) / N$} & $N$ & \multicolumn{2}{|c|}{$\left(\log _{2} N\right) / N$} \\
\hline & 88 & \multicolumn{2}{|c|}{0.07340} & 183 & \multicolumn{2}{|c|}{0.04107} & 153 & \multicolumn{2}{|c|}{0.04743} \\
\hline \multirow[t]{2}{*}{ Diameter } & \multicolumn{3}{|c|}{6} & \multicolumn{3}{|c|}{4} & \multicolumn{3}{|c|}{6} \\
\hline & $P_{\delta}(h)$ & $C(h)$ & $P_{\delta}(h) C(h)$ & $P_{\delta}(h)$ & $C(h)$ & $P_{\delta}(h) C(h)$ & $P_{\delta}(h)$ & $C(h)$ & $P_{\delta}(h) C(h)$ \\
\hline$h=1$ & 0.03579 & 0.6097 & 0.021821 & 0.14616 & 0.3281 & 0.04796 & 0.03139 & 0.5019 & 0.01575 \\
\hline$h=2$ & 0.11834 & 0.1044 & 0.012355 & 0.56266 & 0.1217 & 0.06848 & 0.17011 & 0.0845 & 0.01437 \\
\hline$h=3$ & 0.20977 & 0.0789 & 0.016551 & 0.28956 & 0.0970 & 0.02809 & 0.26049 & 0.0592 & 0.01542 \\
\hline$h=4$ & 0.26829 & 0.0231 & 0.006197 & 0.00162 & 0.9670 & 0.00157 & 0.45700 & 0.0064 & 0.00292 \\
\hline$h=5$ & 0.18939 & 0.0410 & 0.007765 & & & & 0.07843 & 0.0001 & 7.8E-6 \\
\hline$h=6$ & 0.17842 & 0.0617 & 0.011009 & & & & 0.00258 & 0 & 0 \\
\hline
\end{tabular}

\begin{tabular}{|c|c|c|c|c|c|c|c|c|c|}
\hline Network & \multicolumn{3}{|c|}{ ythan } & \multicolumn{3}{|c|}{ elegans } & \multicolumn{3}{|c|}{ helico } \\
\hline \multirow{2}{*}{ Vertices } & $N$ & \multicolumn{2}{|c|}{$\left(\log _{2} N\right) / N$} & $N$ & \multicolumn{2}{|c|}{$\left(\log _{2} N\right) / N$} & $N$ & \multicolumn{2}{|c|}{$\left(\log _{2} N\right) / N$} \\
\hline & 153 & \multicolumn{2}{|c|}{0.04743} & 314 & \multicolumn{2}{|c|}{0.02642} & 710 & \multicolumn{2}{|c|}{0.01334} \\
\hline \multirow[t]{2}{*}{ Diameter } & \multicolumn{3}{|c|}{4} & \multicolumn{3}{|c|}{15} & \multicolumn{3}{|c|}{9} \\
\hline & $P_{\delta}(h)$ & $C(h)$ & $P_{\delta}(h) C(h)$ & $P_{\delta}(h)$ & $C(h)$ & $P_{\delta}(h) C(h)$ & $P_{\delta}(h)$ & $C(h)$ & $P_{\delta}(h) C(h)$ \\
\hline$h=1$ & 0.06589 & 0.2675 & 0.01763 & 0.00739 & 0.6689 & 0.00494 & 0.00555 & 0.3915 & 0.00217 \\
\hline$h=2$ & 0.48292 & 0.0960 & 0.04636 & 0.04143 & 0.0530 & 0.00220 & 0.05246 & 0.0282 & 0.00148 \\
\hline$h=3$ & 0.42300 & 0.0249 & 0.01053 & 0.04092 & 0.0773 & 0.00316 & 0.18910 & 0.0190 & 0.00359 \\
\hline
\end{tabular}




\begin{tabular}{c|l|l|l|l|l|l|l|l|l}
\hline$h=4$ & 0.02819 & 0.1804 & 0.00509 & 0.13101 & 0.0350 & 0.00459 & 0.40280 & 0.0048 & 0.00193 \\
\hline$h=5$ & & & & 0.10260 & 0.0271 & 0.00278 & 0.25290 & 0.0017 & 0.00043 \\
\hline$h=6$ & & & & 0.16432 & 0.0044 & 0.00072 & 0.08311 & 0.0116 & 0.00096 \\
\hline$h=7$ & & & & 0.13419 & 0.0056 & 0.00075 & 0.01264 & 0.3659 & 0.00462 \\
\hline$h=8$ & & & & 0.13970 & 0.0029 & 0.00041 & 0.00139 & 0.6276 & 0.00087 \\
\hline$h=9$ & & & & 0.09574 & 0.0016 & 0.00015 & 0.00006 & 0.8235 & 0.00005 \\
\hline$h=10$ & & & & 0.07511 & 0.0007 & 0.00005 & & & \\
\hline$h=11$ & & & & 0.03763 & 0.0003 & 0.00001 & & & \\
\hline$h=12$ & & & & 0.02151 & 0.0001 & $2.2 \mathrm{E}-6$ & & & \\
\hline$h=13$ & & & & 0.00580 & $9.9 \mathrm{E}-5$ & $5.7 \mathrm{E}-7$ & & & \\
\hline$h=14$ & & & & 0.00244 & 0 & 0 & & & \\
\hline$h=15$ & & & & 0.00020 & 0 & 0 & & & \\
\hline
\end{tabular}

\begin{tabular}{|c|c|c|c|c|c|c|c|c|c|}
\hline Network & \multicolumn{3}{|c|}{ linux } & \multicolumn{3}{|c|}{ abiword } & \multicolumn{3}{|c|}{ mysql } \\
\hline \multirow{2}{*}{ Vertices } & $N$ & \multicolumn{2}{|c|}{$\left(\log _{2} N\right) / N$} & $N$ & \multicolumn{2}{|c|}{$\left(\log _{2} N\right) / N$} & $N$ & \multicolumn{2}{|c|}{$\left(\log _{2} N\right) / N$} \\
\hline & 5285 & \multicolumn{2}{|c|}{0.00234} & 1035 & \multicolumn{2}{|c|}{0.00967} & 1480 & \multicolumn{2}{|c|}{0.00712} \\
\hline \multirow[t]{2}{*}{ Diameter } & \multicolumn{3}{|c|}{17} & \multicolumn{3}{|c|}{12} & \multicolumn{3}{|c|}{16} \\
\hline & $P_{\delta}(h)$ & $C(h)$ & $P_{\delta}(h) C(h)$ & $P_{\delta}(h)$ & $C(h)$ & $P_{\delta}(h) C(h)$ & $P_{\delta}(h)$ & $C(h)$ & $P_{\delta}(h) C(h)$ \\
\hline$h=1$ & 0.00081 & 0.33301 & 0.00027 & 0.00321 & 0.4901 & 0.00157 & 0.00383 & 0.3305 & 0.00126 \\
\hline$h=2$ & 0.04767 & 0.06361 & 0.00303 & 0.02709 & 0.0669 & 0.00181 & 0.06244 & 0.0796 & 0.00497 \\
\hline$h=3$ & 0.15141 & 0.01921 & 0.00291 & 0.10691 & 0.0307 & 0.00328 & 0.18590 & 0.0243 & 0.00452 \\
\hline$h=4$ & 0.27863 & 0.00465 & 0.0013 & 0.23474 & 0.0089 & 0.00290 & 0.19607 & 0.0134 & 0.00263 \\
\hline$h=5$ & 0.27115 & 0.00130 & 0.00035 & 0.24933 & 0.0038 & 0.00095 & 0.11787 & 0.0110 & 0.00130 \\
\hline$h=6$ & 0.156678 & 0.00079 & 0.00012 & 0.21003 & 0.0020 & 0.00042 & 0.08641 & 0.0118 & 0.00102 \\
\hline$h=7$ & 0.06248 & 0.00126 & $7.87 \mathrm{E}-05$ & 0.10807 & 0.0462 & 0.00499 & 0.10237 & 0.0143 & 0.00146 \\
\hline$h=8$ & 0.02079 & 0.00152 & $3.17 \mathrm{E}-05$ & 0.04598 & 0.2120 & 0.00974 & 0.11104 & 0.0106 & 0.00118 \\
\hline$h=9$ & 0.00712 & 0.01979 & 0.00014 & 0.01263 & 0.2861 & 0.00361 & 0.07841 & 0.0077 & 0.00060 \\
\hline$h=10$ & 0.00233 & 0.26923 & 0.00063 & 0.00196 & 0.2681 & 0.00053 & 0.03580 & 0.0182 & 0.00065 \\
\hline$h=11$ & 0.00069 & 0.52121 & 0.00036 & 0.00004 & 0.9290 & 0.00004 & 0.01352 & 0.0268 & 0.00036 \\
\hline$h=12$ & 0.00018 & 0.63878 & 0.00011 & $1.9 \mathrm{E}-6$ & 1 & $1.9 \mathrm{E}-6$ & 0.00474 & 0.3974 & 0.00188 \\
\hline$h=13$ & $3.46 \mathrm{E}-5$ & 0.61215 & $2.12 \mathrm{E}-5$ & & & & 0.00128 & 0.6713 & 0.00086 \\
\hline$h=14$ & $9.17 \mathrm{E}-6$ & 0.5776 & $5.29 \mathrm{E}-6$ & & & & 0.00028 & 0.7133 & 0.00020 \\
\hline$h=15$ & $1.43 \mathrm{E}-6$ & 0.81818 & $1.17 \mathrm{E}-6$ & & & & 0.00004 & 0.8097 & 0.00003 \\
\hline$h=16$ & $4.3 \mathrm{E}-7$ & 0.875 & $3.76 \mathrm{E}-7$ & & & & $1.8 \mathrm{E}-6$ & 0.6667 & $1.2 \mathrm{E}-6$ \\
\hline$h=17$ & $7.16 \mathrm{E}-8$ & 1 & 7.16E-8 & & & & & & \\
\hline
\end{tabular}




\begin{tabular}{|c|c|c|c|c|c|c|c|c|c|}
\hline Networks & \multicolumn{3}{|c|}{$\mathrm{xmms}$} & \multicolumn{3}{|c|}{ vtk } & \multicolumn{3}{|c|}{ NCSTRL } \\
\hline \multirow{2}{*}{ Vertices } & $N$ & \multicolumn{2}{|c|}{$\left(\log _{2} N\right) / N$} & $N$ & \multicolumn{2}{|c|}{$\left(\log _{2} N\right) / N$} & $N$ & \multicolumn{2}{|c|}{$\left(\log _{2} N\right) / N$} \\
\hline & 971 & \multicolumn{2}{|c|}{0.01022} & 771 & \multicolumn{2}{|c|}{0.01244} & 6396 & \multicolumn{2}{|c|}{0.00198} \\
\hline \multirow[t]{2}{*}{ Diameter } & \multicolumn{3}{|c|}{17} & \multicolumn{3}{|c|}{11} & \multicolumn{3}{|c|}{31} \\
\hline & $P_{\delta}(h)$ & $C(h)$ & $P_{\delta}(h) C(h)$ & $P_{\delta}(h)$ & $C(h)$ & $P_{\delta}(h) C(h)$ & $P_{\delta}(h)$ & $C(h)$ & $P_{\delta}(h) C(h)$ \\
\hline$h=1$ & 0.00383 & 0.3637 & 0.00139 & 0.00457 & 0.5124 & 0.00234 & 0.00078 & 0.79621 & 0.00062 \\
\hline$h=2$ & 0.02105 & 0.0740 & 0.00156 & 0.04686 & 0.0676 & 0.00317 & 0.00284 & 0.25672 & 0.00073 \\
\hline$h=3$ & 0.06371 & 0.0355 & 0.00226 & 0.16215 & 0.0178 & 0.00289 & 0.00547 & 0.17345 & 0.00095 \\
\hline$h=4$ & 0.13053 & 0.0125 & 0.00163 & 0.28570 & 0.0098 & 0.00280 & 0.01170 & 0.10203 & 0.00119 \\
\hline$h=5$ & 0.16721 & 0.0067 & 0.00112 & 0.27918 & 0.0025 & 0.00070 & 0.02383 & 0.06241 & 0.00149 \\
\hline$h=6$ & 0.16653 & 0.0049 & 0.00082 & 0.16089 & 0.0011 & 0.00018 & 0.04506 & 0.03802 & 0.00171 \\
\hline$h=7$ & 0.15524 & 0.0050 & 0.00078 & 0.04758 & 0.0111 & 0.00053 & 0.07529 & 0.02076 & 0.00156 \\
\hline$h=8$ & 0.12190 & 0.0056 & 0.00068 & 0.01116 & 0.0253 & 0.00028 & 0.10631 & 0.01397 & 0.00149 \\
\hline$h=9$ & 0.08075 & 0.0069 & 0.00056 & 0.00176 & 0.0180 & 0.00003 & 0.12810 & 0.00909 & 0.00116 \\
\hline$h=10$ & 0.04634 & 0.0370 & 0.00171 & 0.00013 & 0.1739 & 0.00002 & 0.13281 & 0.00703 & 0.00093 \\
\hline$h=11$ & 0.02444 & 0.1720 & 0.00420 & $6.7 \mathrm{E}-6$ & 0.6667 & 4.4E-6 & 0.12287 & 0.00594 & 0.00073 \\
\hline$h=12$ & 0.01124 & 0.3492 & 0.00393 & & & & 0.10300 & 0.00539 & 0.00055 \\
\hline$h=13$ & 0.00479 & 0.3788 & 0.00181 & & & & 0.08012 & 0.00711 & 0.00057 \\
\hline$h=14$ & 0.00171 & 0.4487 & 0.00077 & & & & 0.05759 & 0.01043 & 0.00060 \\
\hline$h=15$ & 0.00055 & 0.4854 & 0.00027 & & & & 0.03874 & 0.02055 & 0.00080 \\
\hline$h=16$ & 0.00016 & 0.4614 & 0.00007 & & & & 0.02526 & 0.04302 & 0.00109 \\
\hline$h=17$ & 0.00001 & 0.8333 & 8.3E-6 & & & & 0.01581 & 0.09419 & 0.00149 \\
\hline$h=18$ & & & & & & & 0.00992 & 0.18115 & 0.00180 \\
\hline$h=19$ & & & & & & & 0.00610 & 0.31991 & 0.00195 \\
\hline$h=20$ & & & & & & & 0.00371 & 0.45606 & 0.00169 \\
\hline$h=21$ & & & & & & & 0.00216 & 0.57063 & 0.00123 \\
\hline$h=22$ & & & & & & & 0.00123 & 0.65030 & 0.00080 \\
\hline$h=23$ & & & & & & & 0.00067 & 0.70962 & 0.00047 \\
\hline$h=24$ & & & & & & & 0.00033 & 0.77422 & 0.00025 \\
\hline$h=25$ & & & & & & & 0.00017 & 0.80562 & 0.00014 \\
\hline$h=26$ & & & & & & & $7.28 \mathrm{E}-5$ & 0.80565 & $5.87 \mathrm{E}-5$ \\
\hline$h=27$ & & & & & & & $3.97 \mathrm{E}-5$ & 0.81525 & 3.23E-5 \\
\hline$h=28$ & & & & & & & $1.61 \mathrm{E}-5$ & 0.82742 & $1.34 \mathrm{E}-5$ \\
\hline$h=29$ & & & & & & & $5.28 \mathrm{E}-6$ & 0.88391 & 4.67E-6 \\
\hline$h=30$ & & & & & & & $1.37 \mathrm{E}-6$ & 0.93998 & $1.29 \mathrm{E}-6$ \\
\hline$h=31$ & & & & & & & $1.96 \mathrm{E}-7$ & 0.83333 & $1.63 \mathrm{E}-7$ \\
\hline
\end{tabular}




\section{References}

[1] Akers S.B. \& Krishnamurthy, B. (1989). A group theoretic model for symmetric interconnection networks. IEEE Transactions on Computers, 38(4):555-566

[2] Barabási, A.-L. \& Albert, R. (1999). Emergence of scaling in random networks. Science, 286: 509-512

[3] Biggs, N. (1993). Algebraic Graph Theory. Cambridge Univ. Press

[4] Kim, B.J., Yoon, C.N., Han, S.K., \& Jeong, H. (2002). Path finding strategies in scale-free networks. Physical Review E, 65: 027103

[5] Milgram, S. (1967). The small world problem. Psychology Today, 1: 61-67

[6] Montoya, J.M. \& Sole, R.V. (2002). Small world patterns in food webs. Journal of Theoretical Biology, 214: 405-412

[7] Myers, C.R. (2003). Software systems as complex networks: Structure, function, and evolvability of software collaboration graphs. Physical Review E, 68: 046116

[8] Newman, M.E.J. (2001). Scientific collaboration networks. I. Network construction and fundamental results. Physical Review E, 64: 016131

[9] Newman, M.E.J. (2003). The structure and function of complex networks. SIAM Review, 45: 167-256

[10]Watts, D.J. \& Strogatz, S.H. (1998). Collective dynamics of 'small-world' networks. Nature, 393: 440-442

[11]Xiao, W.J. \& Parhami, B. (2006). Cayley graphs as models of deterministic small-world networks. Information Processing Letters, 97: 115-117

[12]Xiao, W.J. \& Parhami, B. (2005). On conditions for scale-freedom in complex networks. Working Paper

Wenjun Xiao received the Ph.D degree in mathematics from Sichuan University, People's Republic of China, in 1989. Currently, he is a professor in the School of Computer Science and Engineering, South China University of Technology, Guangzhou, People's Republic of China. His research interests include discrete mathematics, parallel and distributed computing, complex networks, and software architecture. $\mathrm{He}$ has published more than 50 papers in journals and conferences on these topics since 1985.

Wenhong Wei, Weidong Chen and Yong Qin are $\mathrm{Ph} . \mathrm{D}$ candidates in the School of Computer Science and Engineering, South China University of Technology, Guangzhou, People's Republic of China. Their research interests include parallel and distributed computing and networks.

Behrooz Parhami received the Ph.D degree in computer science from the University of California, Los Angeles, in 1973. Presently, he is a professor in the Department of Electrical and Computer Engineering, University of California, Santa Barbara. His research deals with parallel architectures and algorithms, computer arithmetic, and reliable computing. In his previous position with Sharif University of Technology in Tehran, Iran (1974-1988), he was also involved in the areas of educational planning, curriculum development, standardization efforts, technology transfer, and various editorial responsibilities, including a 
five-year term as editor of Computer Report, a Farsilanguage computing periodical. Dr. Parhami's technical publications include more than 220 papers in journals and international conferences, a Farsi-language textbook, and an English/Farsi glossary of computing terms. Among his latest publications are two graduate-level textbooks on parallel processing (Plenum, 1999) and computer arithmetic (Oxford, 2000), and an introductory textbook on computer architecture (Oxford, 2005). Dr. Parhami is a fellow the IEEE and the IEEE Computer Society, a chartered fellow of the British Computer Society, a member of the $\mathrm{ACM}$, and a distinguished member of the Informatics Society of Iran, for which he served as a founding member and president from 1979-1984. He also served as chairman of the IEEE Iran Section (1977-1986) and received the IEEE Centennial Medal in 1984. 